\title{
In vivo/ex vivo targeting of Langerhans cells after topical application of the immune response modifier TMX-202: confocal Raman microscopy and histology analysis
}

Maxim E. Darvin

Gisela Thiede

Saul Mujica Ascencio

Sabine Schanzer

Heike Richter

Sabrina E. Vinzón

Daniel Hasche

Frank Rösl

Roberto May

Yohan Hazot

Dov Tamarkin

Juergen Lademann 


\title{
In vivolex vivo targeting of Langerhans cells after topical application of the immune response modifier TMX-202: confocal Raman microscopy and histology analysis
}

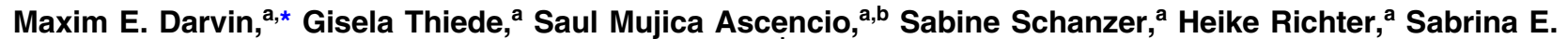 \\ Vinzón,, ${ }^{c}$ Daniel Hasche, ${ }^{c}$ Frank Rösl, ${ }^{c}$ Roberto May, ${ }^{d}$ Yohan Hazot, ${ }^{e}$ Dov Tamarkin, ${ }^{e}$ and Juergen Lademann ${ }^{a}$ \\ ${ }^{a}$ Charité-Universitätsmedizin Berlin, Department of Dermatology, Venerology, and Allergology, Charitéplatz 1, 10117 Berlin, Germany \\ ${ }^{\text {b} C e n t r o ~ d e ~ I n v e s t i g a c i o ́ n ~ e ~ I n n o v a c i o ́ n ~ T e c n o l o ́ g i c a ~(C I I T E C) ~ d e l ~ I n s t i t u t o ~ P o l i t e ́ c n i c o ~ N a c i o n a l ~(I P N), ~ C e r r a d a ~ d e ~ C e c a t i ~ S / N, ~}$ \\ Col. Santa Catarina, Azcapotzalco, CP: 02250 México D. F., Mexico \\ 'DKFZ—Division of Viral Transformation Mechanisms, German Cancer Research Center, Im Neuenheimer Feld 280, Heidelberg 69120, Germany \\ ${ }^{\mathrm{d}}$ Telormedix S.A, Via Lugano 13, Agno CH-6982, Switzerland \\ ${ }^{\mathrm{e}}$ Foamix Pharmaceuticals Ltd., 2 Holzman Street, Weizmann-Science Park, Rehovot 7670402, Israel
}

\begin{abstract}
The increased ability of TMX-202 (derivative of imiquimod) to penetrate the intact stratum corneum (SC) and the follicular orifices of porcine ear skin was shown ex vivo using confocal Raman microscopy and laser scanning microscopy. Moreover, to assess whether TMX-202 is able to reach the immune cells, Langerhans cells extracted from pretreated human skin were investigated ex vivo using confocal Raman microscopy combined with multivariate statistical methods. Tracking the Raman peak of dimethyl sulfoxide centered at $690 \mathrm{~cm}^{-1}$, the absorption of TMX-202 containing formulation by Langerhans cells was shown. To answer the question whether the TMX-202 active ingredient is able to reach Langerhans cells, the attraction of immune cells to TMX-202 containing formulation treated skin was measured in the in vivo rodent model Mastomys coucha. The results show that TMX-202 active ingredient is able to reach Langerhans cells after penetrating through the intact skin and subsequently attract immune cells. Both the intercellular/transcellular as well as the follicular pathways allow the penetration through the intact barrier of the SC. ๑ 2016 Society of Photo-Optical Instrumentation Engineers (SPIE) [DOI: 10.1117/1.JBO.21.5.055004]
\end{abstract}

Keywords: principal component analysis-linear discriminant analysis method; low concentration; traces; dimethyl sulfoxide; immune cells; Raman spectrum of Langerhans cells; Raman spectra analysis.

Paper 150855R received Dec. 21, 2015; accepted for publication May 3, 2016; published online May 27, 2016.

\section{Introduction}

Toll receptor 7 (TLR7) has emerged as a particularly important and unique target for the development of drugs that modulate the innate immune system as it binds not only naturally occurring single stranded RNA but also synthetic low molecular weight ligands with classical drug-like properties, such as imiquimod (an imidazoquinoline) and purine-like molecules. ${ }^{1,2}$ Acting as an immune response modifier, imiquimod is widely used as a topical agent in dermatology for the treatment of various skin diseases, such as actinic keratosis and nonmelanoma skin cancer, $^{3-5}$ which in humans are known to be linked to UV exposure, immunosuppression, and papillomavirus infection. ${ }^{6,7}$ Imiquimod and its derivatives modulate innate immune responses by binding to TLR7 in dendritic and Langerhans cells, monocytes, and macrophages to induce the synthesis of IFN- $\alpha$ and proinflammatory Th 1 cytokines. ${ }^{8}$

Therefore, the topically applied immune response modifier must be able to overcome the cutaneous barrier in order to reach their targets and exert their function. Enhancers of penetration are important components of cosmetic and medical formulations, thus enabling active substances to enter easier through

*Address all correspondence to: Maxim E. Darvin, E-mail: maxim.darvin@ charite.de the cutaneous barrier. ${ }^{9,10}$ Passive penetration of imiquimod through the skin barrier is negligible; therefore, its combination with an enhancing carrier is an important step toward improved clinical efficacy. TMX-202 is a derivative of imiquimod with lesser systemic toxicity, prepared by conjugating to a $\mathrm{C}-12$ phospholipid via a versatile benzoic acid functional group. ${ }^{3}$ This modification should enhance the penetration of the formulation through the skin barrier facilitating its absorption by the living cells of the stratum granulosum and stratum spinosum, including Langerhans cells. Local application of TMX-202 activates the TLR7 receptor in the immune cells, leading to their attraction to the skin.

The different routes, i.e., directly through the stratum corneum (SC) and into the hair follicles, were shown to take a part in the penetration processes. ${ }^{11}$ To test whether the TMX202 is able to penetrate through the SC barrier and to reach the Langerhans cells, confocal Raman microscopic measurements were performed ex vivo on human skin samples pretreated with a TMX-202 containing formulation, and on Langerhans cells extracted therefrom. The skin samples were obtained from healthy patients undergoing abdominal reduction esthetic surgery. Additionally, the biologic effect of TMX-202, namely the attraction of immune cells, was shown in vivo in the rodent

$1083-3668 / 2016 / \$ 25.00$ @ 2016 SPIE 
Mastomys coucha, a unique model for papilloma virus-induced skin carcinogenesis. ${ }^{12}$

Since very low concentrations of TMX-202 containing formulation were expected to penetrate through the SC barrier and reach the Langerhans cells, the measuring technique, the excitation conditions as well as analysis procedure had to be optimized for detection of trace concentrations of TMX-202 containing formulation.

The aim of the present study was to prove whether the TMX202 topically applied on the skin is able to reach and influence Langerhans cells, finally exerting an immune modulatory effect.

\section{Materials and Method}

\subsection{Investigated Substance}

A TMX-202 containing foam formulation was used in the present study for topical application. The active ingredient TMX-202 (2-(4-\{[6-Amino-2-(2-methoxyethoxy)-8-oxo-7Hpurin-9(8H)-yl]methyl \}benzamido)ethyl2,3-Bis (dodecanoyloxy) propyl phosphate) was provided by Telormedix S.A. (Bioggio, Switzerland) which subcontracted the synthesis to Corden Pharma Switzerland LCC (Liestal, Switzerland). The most concentrated ingredients of the utilized foam formulation were dimethyl sulfoxide (45\%), oleic acid $(25 \%)$, polyethylene glycol 200 (16\%), and the active ingredient TMX-202 (5\%). The utilized foam formulation was developed and manufactured by Foamix Pharmaceuticals Ltd., Rehovot, Israel.

\subsection{Skin Samples}

The study was performed on both human and animal skin samples. The human skin was obtained immediately after esthetic abdominal reduction surgery of healthy patients at the Charite-Universitätsmedizin hospital. Subsequently, the excised human skin was pretreated with TMX-202 containing formulation for $1 \mathrm{~h}$. Subsequently, the Langerhans cells were extracted.

Porcine ear skin samples were obtained from a local abattoir in Berlin. The ears were gently cleaned with cold flowing tap water and dried with soft tissue. Hairs were removed without damaging the SC. Thereafter, the porcine ear skin samples were pretreated with TMX-202 containing formulation for $1 \mathrm{~h}$. Porcine skin was chosen for the measurements due to its similarity with human skin and appropriateness for ex vivo measurements. ${ }^{13,14}$

Mastomys coucha from the DKFZ breeding colony were maintained under conventional conditions described elsewhere. ${ }^{12}$ The animals were treated fewer than $3 \%$ Isoflurane anesthesia to allow penetration of the compound and to minimize suffering of the animal. The animals were shaved at the back $(2 \times 2 \mathrm{~cm})$ and treated twice per week with a foam containing 5\% TMX-202 or a placebo foam $(n=5)$. After 12 weeks, the animals were sacrificed and skin biopsies were taken for histological analysis.

Skin sample areas without any scars and visible defects were chosen for topical application of the TMX-202 containing formulation.

\subsection{Langerhans Cells Extraction}

The human skin was obtained after abdominal reduction esthetic surgery of healthy patients immediately after surgery. The Langerhans cells were extracted from the epidermis of six patients following the procedure described elsewhere. ${ }^{15,16}$ The extracted Langerhans cells were diluted in paraformaldehyde (PFA) and stored at $+4^{\circ} \mathrm{C}$.

\subsection{Confocal Raman Microscopy}

Two different commercially available CRM- "Horiba" (model Labram HR800 Evo, France, excitation wavelengths: 325, 473, and $633 \mathrm{~nm}$ ) and "River Diagnostics" (model 3510 SCA, Rotterdam, Netherlands, excitation wavelength $785 \mathrm{~nm}$ ) were used for the determination of TMX-202 containing formulation in the Langerhans cells. Only the fingerprint spectral range (400 to $1800 \mathrm{~cm}^{-1}$ ) was excited and analyzed. The utilized CRMs were described elsewhere. ${ }^{17,18}$

Every Langerhans cell was measured in nine different points in the depth ranging from 0 (surface) to max. $3 \mu \mathrm{m}$ inside the cell. Every Raman spectrum was smoothed with a SavitzkyGolay filter (polynomial order of two and frame size of three) and a baseline correction was employed to subtract the fluorescence background if needed.

\subsection{Determination of the Penetration Depth into the Skin Using Confocal Raman Microscopes}

The penetration depth of the TMX-202 containing formulation into the porcine ear skin was investigated ex vivo using the "Skin Tools" software provided by the company "River Diagnostics." 19 The measurements were performed in the fingerprint range using "River Diagnostics" CRM under the excitation of $785 \mathrm{~nm}$ (maximum $20 \mathrm{~mW}$ on the skin sample). The utilized doses of reference light were not sufficient to influence the skin components. $^{20}$

\subsection{Confocal Laser Scanning Microscopy}

A commercially available confocal laser scanning microscope (VivaScope ${ }^{\circledR} 1500$ Multilaser, Mavig, Germany), which combines reflectance and fluorescent confocal laser scanning microscopy, was used for investigating the histological sections for follicular penetration. The excitation wavelength was $488 \mathrm{~nm}$. A more detailed description of the utilized confocal laser scanning microscope has been published elsewhere. ${ }^{13}$

\subsection{Multivariate Statistical Analysis}

To determine a spectral difference between two groups of Langerhans cells (either treated or not treated with TMX-202 containing formulation) at high sensitivity, multivariate statistical methods such as principal component analysis (PCA) combined with linear discriminant analysis (LDA) were employed. Combination of these statistical methods is widely used in medical practice. ${ }^{21-25}$

PCA is a statistical method that reduces high-dimensional data into lower-dimensional data called principal components (PC). The reconstruction of a Raman spectrum using the first two PCs provides the most relevant spectral information (differences) and disregards less useful information (similarities) from the consideration, thus substantially reducing the noise. $^{26,27}$ The first two PCs are plotted in a two-dimensional (2-D) space in the required spectral range for the TMX-202 treated and untreated Langerhans cells in order to find differences (formulation is present) or similarities (formulation is not present). In order to determine the differences from the reconstructed spectra at high sensitivity, the PCA method is complemented by the LDA method. ${ }^{28-30}$ LDA is considered 
as a group classification as it maximizes the differences between group variances..$^{31,32}$ and reduces the dimension of the data to one dimension. The criterion of the presence of the formulation in the Langerhans cells served the statistically significant differences between the mean values of the two groups, which were calculated using Student's $t$-test $(p<0.05)$, as previously presented. $^{33}$

\subsection{Histological Analysis}

Skin biopsies were fixed in $4 \%$ formaldehyde for $24 \mathrm{~h}$ at room temperature, and embedded in paraffin. Five-micrometer sections were stained with hematoxylin/eosin for histological analysis. Immune cells were counted in three different skin areas.

\subsection{Ethics Statement}

Mastomys coucha at the DKFZ are maintained in compliance with German and European statutes and all animal experiments were approved by local government authorities (Regional Council of Karlsruhe, Germany; G38/13).

Ethical approval for the investigations on excised human skin has been obtained by the local ethics committee of the CharitéUniversitätsmedizin Berlin.

\section{Results and Discussion}

\subsection{Penetration of the TMX-202 Containing Formulation Through the Skin}

The results of the CRM measurements demonstrated that the TMX-202 containing formulation does not penetrate efficiently through the SC. About $90 \%$ was found up to a penetration depth of $\sim 15 \mu \mathrm{m}$. At least $10 \%$ was detectable until a penetration depth of $30 \mu \mathrm{m}$ (Fig. 1), i.e., in the living epidermis.

When analyzing the study results, one should consider the differences between porcine and human skin. According to the literature, the thickness of the human SC is usually between 17 and $28 \mu \mathrm{m} ;{ }^{14}$ however, our experiments were carried out with ears of freshly slaughtered pigs; the thickness of the SC of porcine ear skin was measured between 17 and $20 \mu \mathrm{m}^{34}$ by measuring the water profile. ${ }^{35}$

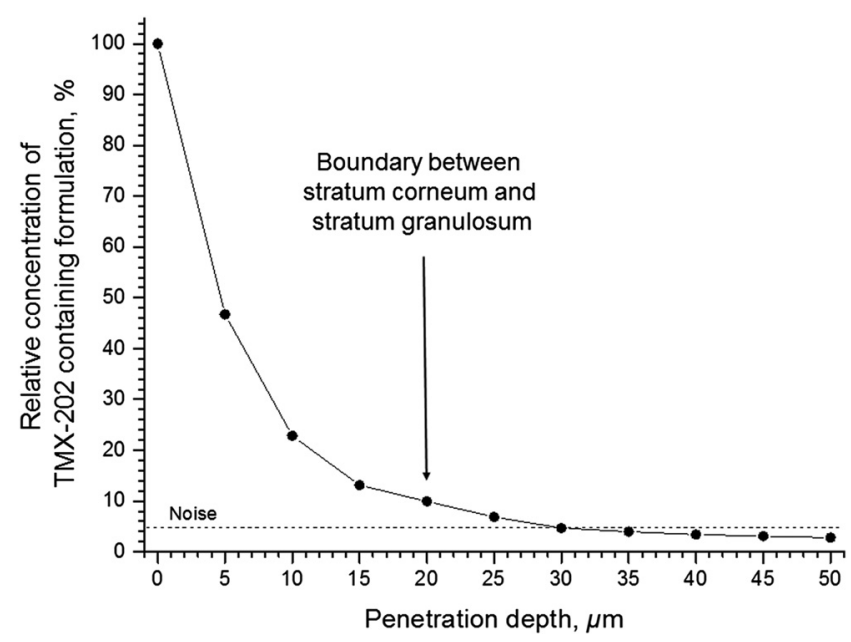

Fig. 1 Relative Raman intensity (normalized to initial value) versus penetration depth for TMX-202 containing formulation (mean value for different six porcine ear skin samples).
The absorption of TMX-202 is very challenging due to its high molecular weight of almost 1000 Dalton. Such molecules usually do not penetrate the skin at all. The fact that high amounts could be detected even in the lower layers of the $\mathrm{SC}$ is encouraging. A penetration into the deeper layers of the $\mathrm{SC}$ is possible, although the penetration time may exceed $24 \mathrm{~h}$.

\subsection{Penetration of TMX-202 Containing Formulation into the Hair Follicles}

Typical results of the penetration of the formulation into the hair follicles are visible in the histological sections. Figure 2 shows a histological section superimposing the fluorescence and reflectance image of the same section measured using laser scanning microscopy. The structure of the hair follicles can be well recognized. The red signal shows the penetration of the TMX-202 containing formulation labeled with sodium fluorescein into the hair follicle.

In Fig. 3, the penetration depths into the hair follicles of six different porcine ears are depicted. At least 10 hair follicles were analyzed for every porcine ear. The mean penetration depth calculated for all six porcine ears was found to be $349 \pm 88 \mu \mathrm{m}$.

Thus, the TMX-202 would be expected to reach the Langerhans cells of the epidermis. In order to study whether this is the case, investigations have to be carried out on human skin. Unfortunately, the follicular penetration is reduced by $90 \%$ ex vivo compared with the in vivo studies. ${ }^{36}$ After surgical excision of the skin, the skin samples contract, partly obstructing the hair follicles. Even, when the skin samples are stretched to their original size, the hair follicles remain partly obstructed. Consequently, if the substance is detectable in Langerhans cells under these ex vivo conditions, the same would be expected to occur in vivo, as the penetration rate under real conditions would be much higher because the hair follicles do not contract in vivo.

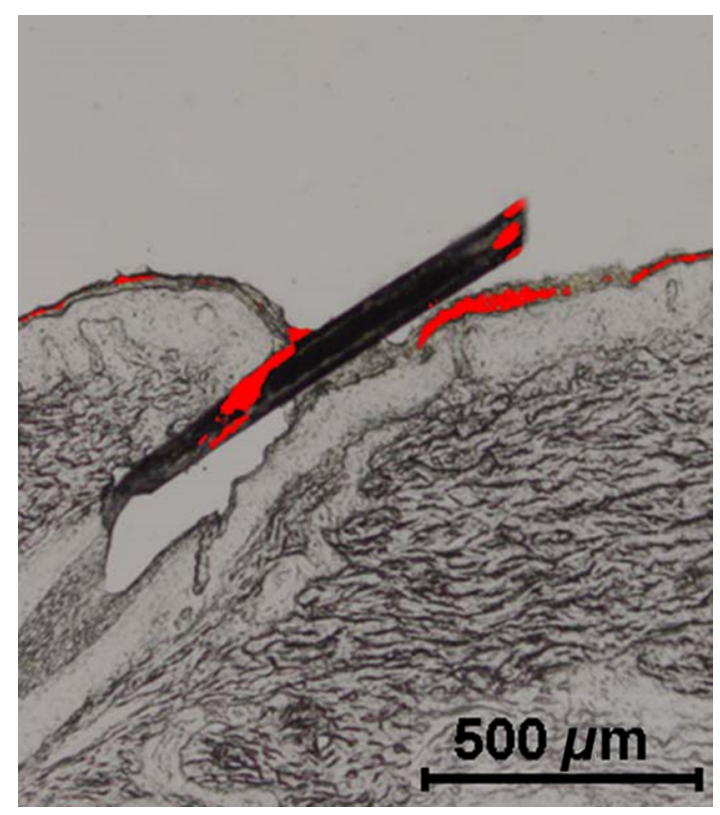

Fig. 2 Representative picture of the penetration of the fluorescencelabeled TMX-202 containing formulation (colored in red) into a hair follicle of porcine skin taken using laser scanning microscopy. 


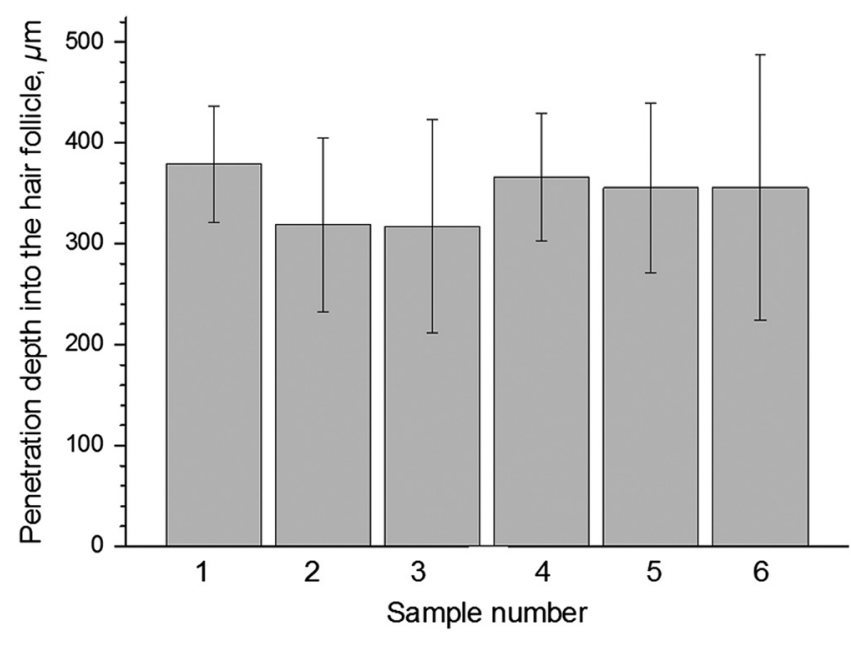

Fig. 3 Penetration depths of the TMX-202 containing formulation into the hair follicles on six different porcine ears (average penetration depth: $349 \pm 88 \mu \mathrm{m}$ ) measured using laser scanning microscopy.

\subsection{Raman Spectroscopy of Langerhans Cells}

Based on the results presented in Secs. 3.1 and 3.2, the TMX202 active ingredient can potentially reach the target immune cells, i.e., the Langerhans cells of the epidermis. In order to prove this anticipation, CRM measurements were performed on Langerhans cells extracted from human skin samples treated with TMX-202 containing formulation.

During the measurements on the extracted cells, three different types of Raman spectra were observed. Figure 4 shows three examples of Raman spectra measured on the extracted cells of one skin sample. The Raman spectra are different and probably originated from different types of cells. The first Raman spectrum (most rarely observed) represents the typical Raman spectrum of keratinocytes or corneocytes [Fig. 4(a)], which is in accordance with already published data. ${ }^{19,37}$ The second Raman spectrum (most frequently observed) has no similarities with keratinocyte or corneocyte Raman spectra [Fig. 4(b)]. The third Raman spectrum shows the superposition of the above-mentioned two spectra [Fig. 4(c)]. The spectra were interpreted as follows: the most frequently observed Raman spectra [Fig. 4(b)] belongs to the Langerhans cells, whereas the rarely observed Raman spectra [Figs. 4(a) and 4(c)] belong to keratinocytes or corneocytes and to a Langerhans cell together with corneocytes or keratinocytes, respectively.
The Raman spectrum shown in Fig. 4(b) was determined to be specific for Langerhans cells for the following reasons: (1) the structure of Langerhans cells is characterized by the presence of cytoplasm, nucleus, and organelles called Birbeck granules, and by the absence of desmosomes and tonofilaments. ${ }^{38,39}$ The desmosomes are responsible for providing a junction between the tonofilaments made mainly by keratin. For this reason the strong Raman peak of keratin, which lies between 1600 and $1700 \mathrm{~cm}^{-1}$, should not be observed in Langerhans cells. (2) The Raman peak centered at $935 \mathrm{~cm}^{-1}$, which represents CC stretching mode of proline, valine, and $\alpha$-helix conformation of protein backbone, ${ }^{40}$ is probably related to the proline-rich protein Langerin, which implicated a Birbeck granule biogenesis in human epidermal Langerhans cells. ${ }^{41}$ (3) After the extraction procedure, the Langerhans cells are dominant in solution, but a low concentration of keratinocytes and probably of corneocytes is also present. ${ }^{15}$ This strongly correlates with the frequency of spectra appearance [Raman spectrum shown in Fig. 4(b) is most frequently observed in comparison to spectra shown in Figs. 4(a) and 4(c)].

As it is impossible to separate Langerhans cells from corneocytes and keratinocytes visually, the cells were screened for the presence of the specific Raman peak at $935 \mathrm{~cm}^{-1}$ before the CRM measurement.

\subsection{Optimization of Excitation Conditions for Confocal Raman Microscopes}

The intensity of Raman signal measured from the dry Langerhans cells (when PFA was evaporated within a few minutes when stored on the glass plate at room conditions) was found to be substantially higher than that measured from the Langerhans cells in PFA solvent. For this reason all measurements were performed on dry samples.

Using two CRM devices operated under the excitation wavelengths of $325,473,633$, and $785 \mathrm{~nm}$, the optimal excitation conditions were found for determining the TMX-202 containing formulation in Langerhans cells using 473-nm excitation wavelength. The blue excitation wavelength at $473-\mathrm{nm}$ gave the highest Raman sensitivity of the TMX-202 containing formulation. Excitation of Langerhans cells also shows that this wavelength can be efficiently used. On the other hand, the Raman spectrum of PFA solvent is "silent" under excitation at $473 \mathrm{~nm}$ showing the absence of Raman and fluorescence signals. Taking all these results into consideration, the blue excitation wavelength at (a)

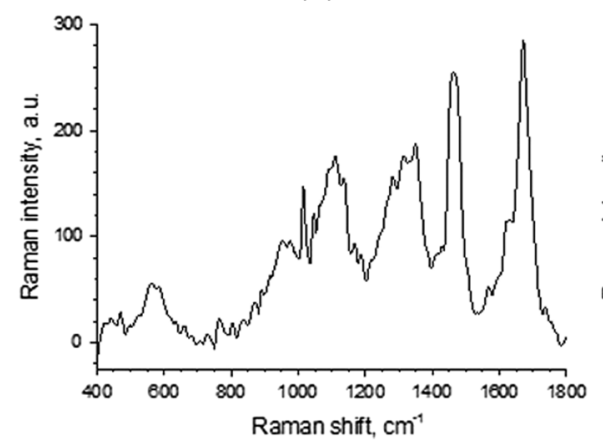

(b)

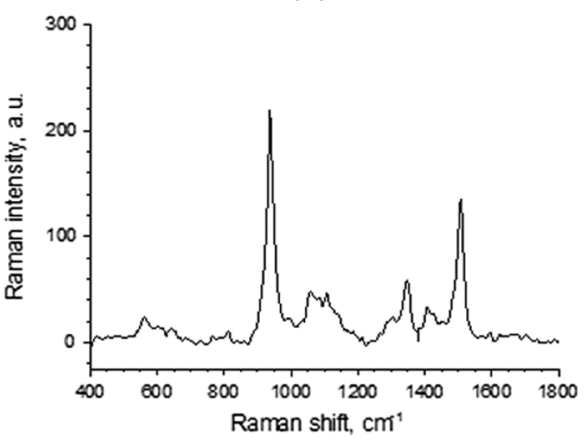

(c)

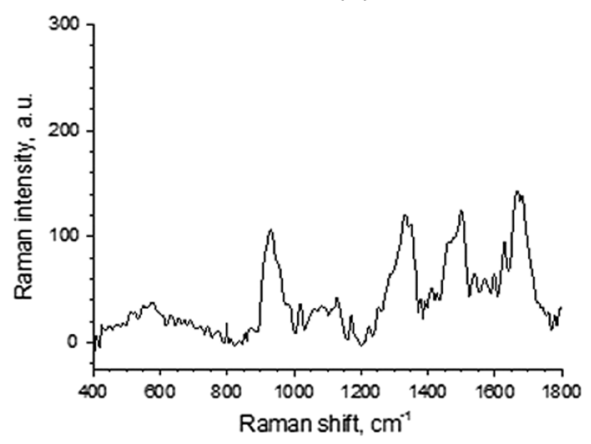

Fig. 4 Raman spectrum of a keratinocyte or corneocyte, (a) a Langerhans cell (b) and a Langerhans cell together with keratinocyte or corneocyte (c) under excitation at $473 \mathrm{~nm}$. 
(a)

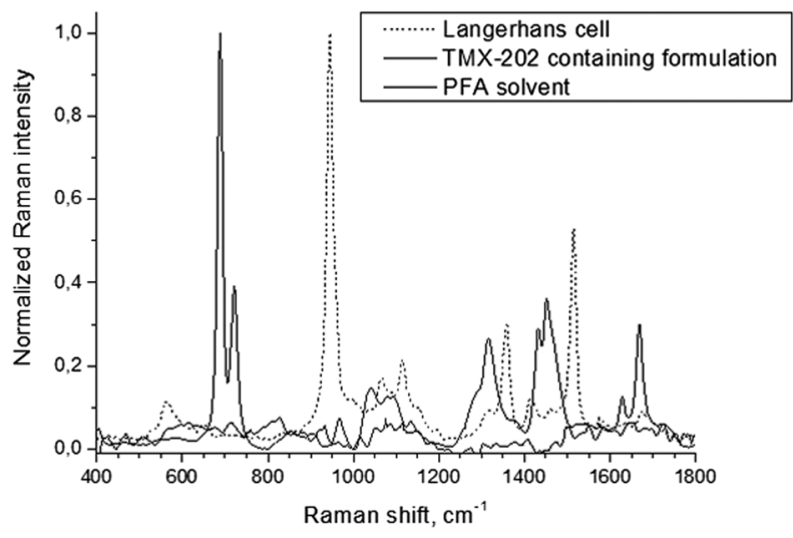

(b)

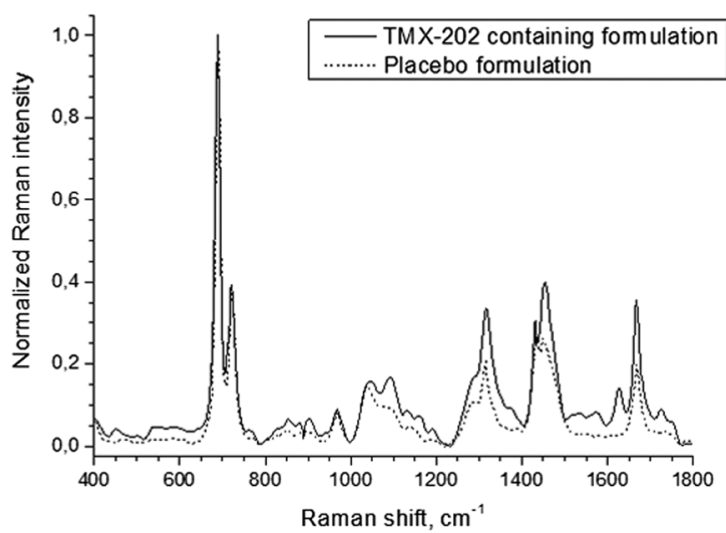

Fig. 5 Raman spectra of TMX-202 containing formulation, Langerhans cells and PFA solvent (a) and Raman spectra of TMX-202 containing and placebo formulations (b) under excitation at $473 \mathrm{~nm}$.

$473 \mathrm{~nm}$ was chosen to be optimal for detection of TMX-202 containing formulation in Langerhans cells. The corresponding spectra are shown in Fig. 5(a).

Moreover, irradiation of Langerhans cells at maximum blue-light power ( $25 \mathrm{~mW}$ on the sample) gave rise to structural changes in the cells detected by the increase of fluorescence background measured post irradiation, which were similar to the spectra measured after irradiation with UV light at $325 \mathrm{~nm}$ ( $3 \mathrm{~mW}$ on the sample). Observed changes are very probably induced by blue-light- and UV-induced free radicals generated in the cells. ${ }^{42,43}$ Therefore, to exclude this effect, the CRM blue-light power was reduced by $50 \%$ to $12.5 \mathrm{~mW}$ on the sample.

\subsection{Determination of TMX-202 Active Ingredient Using Confocal Raman Microscopes}

Figure 5(b) shows Raman spectra obtained under optimal excitation conditions described in Sec. 3.4 for TMX-202 containing and placebo formulations. As can be seen, only a small difference in Raman spectra can be measured at $1626 \mathrm{~cm}^{-1}$ corresponding to N-H vibration, which serves as a characteristic of the TMX-202 active ingredient. The intensity of this peak is very small making impossible the direct detection of TMX-202 active ingredient in the Langerhans cells using CRM. The intensity of the highest peak centered at $690 \mathrm{~cm}^{-1}$, which corresponds to $\mathrm{C}-\mathrm{S}$ vibration of the dimethyl sulfoxide, is $\sim 10$ (a)

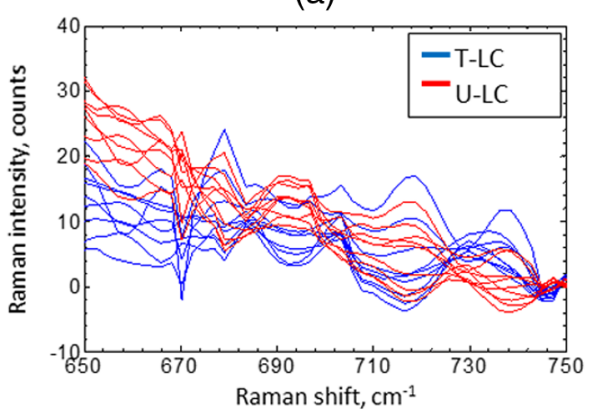

(d)

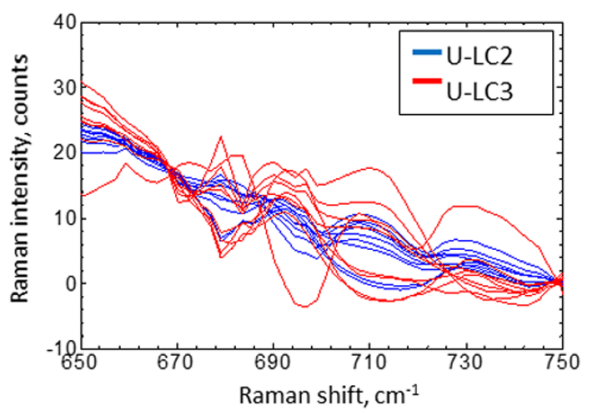

(b)

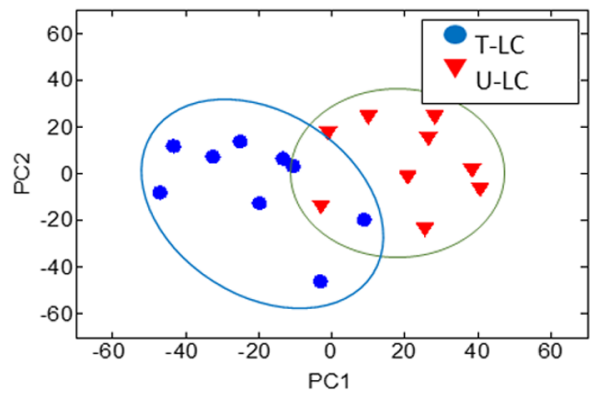

(e)

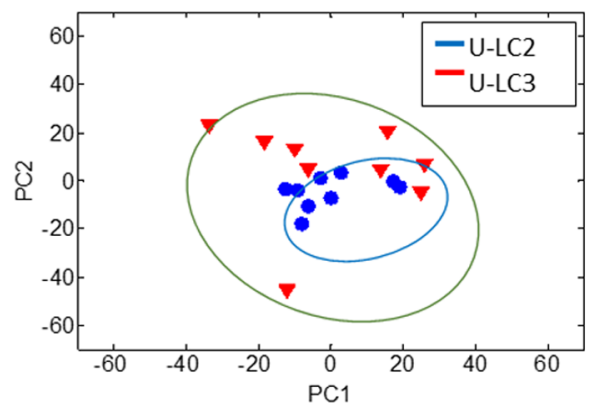

(c)

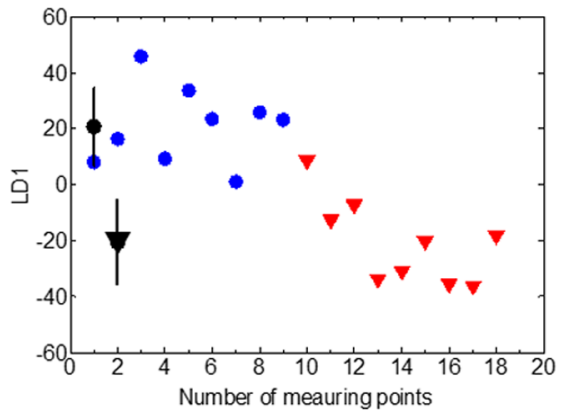

(f)

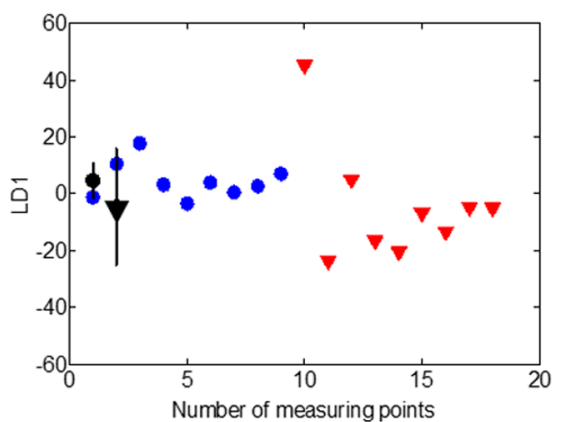

Fig. 6 (a) and (d) Raman spectra of TMX-202 containing formulation treated (T-LC) and untreated (U-LC) Langerhans cells measured in the range of 650 to $750 \mathrm{~cm}^{-1}$ after PCA reconstruction; (b) and (e) 2-D space (PC1; PC2) after the spectra reconstruction; and (c) and (f) PCA-LDA mean values compared using Student's $t$-test $(p<0.05)$. Comparison between (a)-(c) T-LC and U-LC and between (d)-(f) different U-LCs. 
Table 1 Results of multivariate statistical PCA-LDA combined with Student's t-test comparison of TMX-202 containing formulation treated and untreated Langerhans cells. Yes/no-TMX-202 is detected/is not detected in the Langerhans cell. [S1-4: Skin from patient No. 1, skin sample No. 4; LC1(u)—Langerhans cell No. 1 (untreated)].

\begin{tabular}{|c|c|c|c|c|c|c|c|c|}
\hline & S1-4 & S1-4 & S3-1 & S3-1 & S3-1 & S3-2 & S3-3 & S3-3 \\
\hline & LC1 & LC3 & LC1 & LC2 & LC3 & LC1 & LC1 & LC2 \\
\hline S4-1 & Yes & Yes & Yes & Yes & Yes & Yes & Yes & Yes \\
\hline \multicolumn{9}{|l|}{ LC1u } \\
\hline S6-1 & Yes & Yes & Yes & Yes & Yes & Yes & Yes & Yes \\
\hline \multicolumn{9}{|l|}{ LC1u } \\
\hline \multirow{4}{*}{$\begin{array}{l}\text { S6-1 } \\
\text { LC3u }\end{array}$} & Yes & Yes & No & Yes & Yes & Yes & Yes & Yes \\
\hline & & & & & & & & \\
\hline & S3-3 & S5-1 & S5-2 & S6-1 & S6-1 & S6-2 & S6-2 & S6-2 \\
\hline & LC3 & LC2 & LC3 & LC4 & LC5 & LC1 & LC2 & LC4 \\
\hline S4-1 & Yes & Yes & Yes & Yes & Yes & Yes & Yes & No \\
\hline \multicolumn{9}{|l|}{ LC1u } \\
\hline S6-1 & No & Yes & Yes & Yes & Yes & Yes & Yes & Yes \\
\hline \multicolumn{9}{|l|}{ LC1u } \\
\hline S6-1 & No & Yes & Yes & Yes & Yes & Yes & Yes & Yes \\
\hline LC3u & & & & & & & & \\
\hline
\end{tabular}

times higher; therefore, this peak was chosen for determining the TMX-202 containing formulation in the Langerhans cells.

\subsection{Determination of the TMX-202 Containing Formulation in Langerhans Cells}

Figure 5(a) clearly shows that the TMX-202 containing formulation can be efficiently detected in Langerhans cells by tracking the Raman peak centered at $690 \mathrm{~cm}^{-1}$.
Figure 6(a) shows the comparison of example TMX-202 containing formulation treated and untreated Raman spectra of Langerhans cell measured in nine points in the range of 650 to $750 \mathrm{~cm}^{-1}$ after PCA reconstruction, and subsequent 2-D space of the principle components PC1 and PC2 [Fig. 6(b)]. As can be seen from Fig. 6(b), the values inside the overlapped area could not be distinguished. To maximize the differentiation between two groups of data, the complementary statistical method LDA was employed [Fig. 6(c)]. After PCALDA application, the numerical data of two groups could be compared using Student's $t$-test as shown in Fig. 6(c). In order to distinguish the differences between two groups, i.e., to find out whether or not the TMX-202 containing formulation is detectable in Langerhans cells, the $p<0.05$ criteria was chosen. Figures 6(d)-6(f) show the comparison between untreated Langerhans cells. In order to improve the detection of TMX202 containing formulation in Langerhans cells, the untreated Langerhans cells where compared as shown in Figs. 6(d)-6(f). As expected, no differences are visible.

The results are summarized in Table 1 where the groups of data are compared to each other using statistical analyses described earlier. The results show that in most cases (92\%), the TMX-202 containing formulation was measured in the Langerhans cells. It is expected that due to the homogeneous distribution in the foam solvent, the TMX-202 active ingredient will also reach the Langerhans cells.

\subsection{Biological Activity in an Animal Model}

In order to prove whether the TMX-202 active ingredient has reached the Langerhans cells, the histological sections were analyzed. The in vivo effect of the TMX-202 active ingredient was proven in the rodent Mastomys coucha, which represents an excellent animal model of papillomavirus-associated skin disease, thus mimicking the human situation. ${ }^{7,12}$ In the histological analysis of skin treated for 12 weeks, a significantly higher amount of infiltrated immune cells is visible in the epithelia of skin samples treated with TMX-202 containing formulation compared with untreated or placebo treated skin (Fig. 7). Therefore, we could show that the TMX-202 active ingredient is reaching its intended targets in vivo and modulating the skin immune response to further attract immune cells. Furthermore, from the in vivo results it becomes clear that this effect is exerted (a)

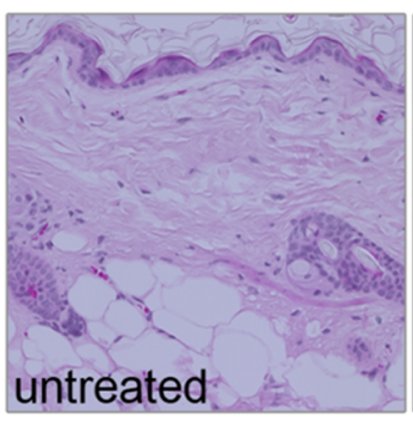

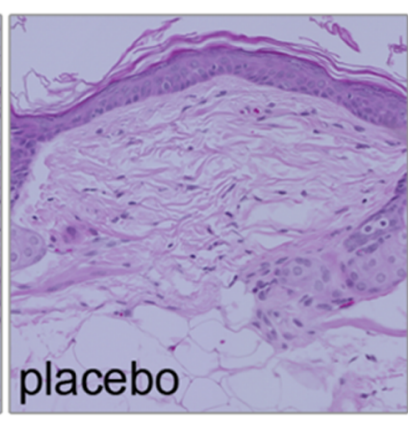

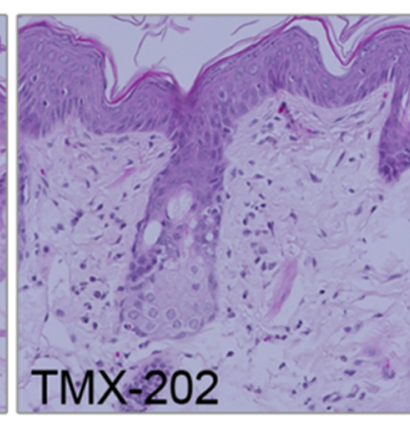

(b)

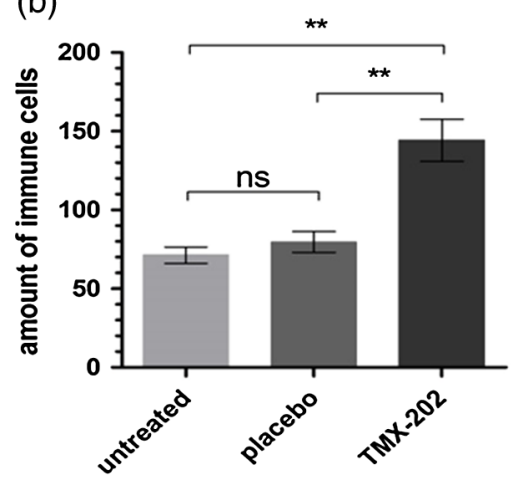

treatment

Fig. 7 (a) Representative areas of skin sections from Mastomys coucha treated with placebo or TMX202 containing formulation for 12 weeks (H\&E staining, magnification 200x) and (b) skin-infiltrating immune cell counts in placebo and TMX-202 containing formulation treated skin. Immune cells in three areas per section were counted $\left({ }^{*} p<0.01\right.$, one way ANOVA). 
by the TMX-202 active ingredient and not the excipients, as the placebo treated skin does not show any difference with respect to the untreated skin.

\section{Conclusions}

The present study shows that the intercellular, transcellular, and follicular pathways are responsible for penetration of TMX-202 containing formulation through the barrier of SC. Reaching living cells of the epidermis, the TMX-202 containing formulation is absorbed in particular by Langerhans cells, measured on human skin samples using CRM combined with multivariate statistical analysis. This method provides information only about the absorption of the formulation containing TMX-202, but not about the absorption of TMX-202 active ingredient by the Langerhans cells. To answer the question whether the TMX-202 active ingredient is able to reach Langerhans cells and exert its biological function, a histological analysis was performed in the skin of the in vivo rodent model Mastomys coucha. The result revealed a significantly higher amount of immune cells infiltrated into the epithelia of TMX-202 containing formulation treated skin samples in comparison to placebo treated and untreated skin samples, which demonstrated TMX202 active ingredient reached the target and induced a strong and local immune activation.

Thus, taking into consideration the limited penetration ability of imiquimod through the skin barrier, the topical application of TMX-202-a derivative of imiquimod containing a penetration enhancer in the form of a phospholipid-is expected to facilitate targeting to cutaneous immune cells.

\section{Acknowledgments}

The described activities have been realized in the frame of TOSCA project (Eurostar E!6872) supported by the Eurostars Programme. Authors would like to thank Mr. Luca Lo Giudice for contribution and support provided during the TOSCA project.

\section{References}

1. H. Hemmi et al., "Small anti-viral compounds activate immune cells via the TLR7 MyD88-dependent signaling pathway," Nat. Immunol. 3(2), 196-200 (2002).

2. J. Lee et al., "Activation of anti-hepatitis $\mathrm{C}$ virus responses via Toll-like receptor 7," Proc. Nat. Acad. Sci. U. S. A. 103(6), 1828-1833 (2006).

3. B. Crain et al., "Inhibition of keratinocyte proliferation by phospholipidconjugates of a TLR7 ligand in a Myc-induced hyperplastic actinic keratosis model in the absence of systemic side effects," Eur. J. Dermatol. 23(5), 618-628 (2013).

4. K. Fields et al., "Localized diffuse melanosis associated with melanoma successfully treated with imiquimod cream 5\%: a case report and review of the literature," Cutis 93(3), 145-150 (2014).

5. N. Kibbi et al., "Treatment of in-transit melanoma with intralesional bacillus calmette-guerin (BCG) and topical imiquimod 5\% cream: a report of 3 cases," J. Immunother. 38(9), 371-375 (2015).

6. M. Tommasino, "The human papillomavirus family and its role in carcinogenesis," Semin. Cancer Biol. 26, 13-21 (2014).

7. S. E. Vinzon and F. Rosl, "HPV vaccination for prevention of skin cancer," Hum. Vaccin. Immunother. 11(2), 353-357 (2015).

8. M. A. Stanley, "Imiquimod and the imidazoquinolones: mechanism of action and therapeutic potential," Clin. Exp. Dermatol. 27(7), 571-577 (2002).

9. M. E. Lane, "Skin penetration enhancers," Int. J. Pharm. 447(1-2), 12-21 (2013).

10. A. C. Williams and B. W. Barry, "Penetration enhancers," Adv. Drug Deliv. Rev. 64, 128-137 (2012).
11. S. Vandersee et al., "Significance of the follicular pathway for dermal substance penetration quantified by laser Doppler flowmetry," J. Biophotonics 9(3), 276-281 (2016).

12. S. E. Vinzon et al., "Protective vaccination against papillomavirusinduced skin tumors under immunocompetent and immunosuppressive conditions: a preclinical study using a natural outbred animal model," PLoS Pathog. 10(2), e1003924 (2014).

13. M. E. Darvin et al., "Comparison of in vivo and ex vivo laser scanning microscopy and multiphoton tomography application for human and porcine skin imaging," Quantum Electron. 44(7), 646-651 (2014).

14. U. Jacobi et al., "Porcine ear skin: an in vitro model for human skin," Skin Res. Technol. 13(1), 19-24 (2007).

15. M. Peiser et al., "CD1 a and CD1c cell sorting yields a homogeneous population of immature human Langerhans cells," J. Immunol. Methods 279(1-2), 41-53 (2003).

16. A. Vogt et al., " $40 \mathrm{~nm}$, but not 750 or $1,500 \mathrm{~nm}$, nanoparticles enter epidermal CD1a+ cells after transcutaneous application on human skin," J. Invest. Dermatol. 126(6), 1316-1322 (2006).

17. F. Bonnier et al., "Analysis of human skin tissue by Raman microspectroscopy: dealing with the background," Vib. Spectrosc. 61, 124-132 (2012).

18. M. E. Darvin et al., "Optical methods for noninvasive determination of carotenoids in human and animal skin," J. Biomed. Opt. 18(6), 061230 (2013).

19. C. Choe, J. Lademann, and M. E. Darvin, "Analysis of human and porcine skin in vivo/ex vivo for penetration of selected oils by confocal Raman microscopy," Skin Pharmacol. Physiol. 28(6), 318-330 (2015).

20. M. Y. Akhalaya et al., "Molecular action mechanisms of solar infrared radiation and heat on human skin," Ageing Res. Rev. 16, 1-11 (2014).

21. L. Chen et al., "Near-infrared confocal micro-Raman spectroscopy combined with PCA-LDA multivariate analysis for detection of esophageal cancer," Laser Phys. 23(6), 065601 (2013).

22. W. W. Chen et al., "Houttuynia cordata Thunb extract induces cytotoxicity in human nasopharyngeal carcinoma cells: Raman spectroscopic studies," Laser Phys. Lett. 13(1), 015601 (2016).

23. S. Feng et al., "Nasopharyngeal cancer detection based on blood plasma surface-enhanced Raman spectroscopy and multivariate analysis," Biosens. Bioelectron. 25(11), 2414-2419 (2010).

24. F. L. Silveira et al., "Discrimination of non-melanoma skin lesions from non-tumor human skin tissues in vivo using Raman spectroscopy and multivariate statistics," Lasers Surg. Med. 47(1), 6-16 (2015).

25. A. C. Terentis et al., "Confocal Raman microspectroscopy discriminates live human metastatic melanoma and skin fibroblast cells," J. Raman Spectrosc. 44(9), 1205-1216 (2013).

26. S. Dochow et al., "Tumour cell identification by means of Raman spectroscopy in combination with optical traps and microfluidic environments," Lab Chip 11(8), 1484-1490 (2011).

27. L. Zhang et al., "Two-stage image denoising by principal component analysis with local pixel grouping," Pattern Recogn. 43(4), 1531-1549 (2010).

28. W. W. Chen et al., "Detection and identification of Huo-Xue-Hua-Yu decoction (HXHYD) using surface-enhanced Raman scattering (SERS) spectroscopy and multivariate analysis," Laser Phys. Lett. 12(4), 045602 (2015).

29. K. Gajjar et al., "Diagnostic segregation of human brain tumours using Fourier-transform infrared and/or Raman spectroscopy coupled with discriminant analysis," Anal. Methods-UK 5(1), 89-102 (2013).

30. C. Sarbu et al., "Classification and fingerprinting of kiwi and pomelo fruits by multivariate analysis of chromatographic and spectroscopic data," Food Chem. 130(4), 994-1002 (2012).

31. A. Subasi and M. I. Gursoy, "EEG signal classification using PCA, ICA, LDA and support vector machines," Expert Syst. Appl. 37(12), 8659-8666 (2010).

32. G. Vitkova et al., "Fast identification of biominerals by means of standoff laser-induced breakdown spectroscopy using linear discriminant analysis and artificial neural networks," Spectrochim. Acta B 73, 1-6 (2012).

33. S. Mujica Ascencio et al., "Confocal Raman microscopy and multivariate statistical analysis for determination of different penetration abilities of caffeine and propylene glycol applied simultaneously in a mixture on porcine skin ex vivo," Eur. J. Pharm. Biopharm. 104, 51-58 (2016). 
34. C. Choe, J. Lademann, and M. E. Darvin, "Confocal Raman microscopy for investigating the penetration of various oils into the human skin in vivo," J. Dermatol. Sci. 79(2), 176-178 (2015).

35. J. M. Crowther et al., "Measuring the effects of topical moisturizers on changes in stratum corneum thickness, water gradients and hydration in vivo," Br. J. Dermatol. 159(3), 567-577 (2008).

36. A. Patzelt et al., "Differential stripping demonstrates a significant reduction of the hair follicle reservoir in vitro compared to in vivo," Eur. J. Pharm. Biopharm. 70(1), 234-238 (2008).

37. Y. Zhu et al., "Penetration of silver nanoparticles into porcine skin ex vivo using fluorescence lifetime imaging microscopy, Raman microscopy, and surface-enhanced Raman scattering microscopy,' J. Biomed. Opt. 20(5), 051006 (2015).

38. S. Jaitley and T. Saraswathi, "Pathophysiology of Langerhans cells," J. Oral Maxillofacial Pathol. 16(2), 239-244 (2012).

39. J. Upadhyay et al., "Langerhans cells and their role in oral mucosal diseases," N. Am. J. Med. Sci. 5(9), 505-514 (2013).
40. Z. Movasaghi, S. Rehman, and I. U. Rehman, "Raman spectroscopy of biological tissues," Appl. Spectrosc. Rev. 42(5), 493-541 (2007).

41. R. Mc Dermott et al., "Birbeck granules are subdomains of endosomal recycling compartment in human epidermal Langerhans cells, which form where Langerin accumulates," Mol. Biol. Cell 13(1), 317-335 (2002).

42. J. Krutmann, "Ultraviolet A radiation-induced biological effects in human skin: relevance for photoaging and photodermatosis," J. Dermatol. Sci. 23(1), S22-S26 (2000)

43. S. Vandersee et al., "Blue-violet light irradiation dose dependently decreases carotenoids in human skin, which indicates the generation of free radicals," Oxidative Med. Cell. Longevity 2015, 579675 (2015).

Biographies for the authors are not available. 